\title{
Biomarkers of natural and vaccine immunity
} against HIV

\begin{abstract}
"...it is increasingly apparent that, as in nature, a protective vaccine immune response will be the result of synergistic and well-regulated interactions ... and therefore a whole profile or biomarker signature based on multiple characteristics will be superior to single biomarkers considered in isolation."
\end{abstract}

\section{KEYWORDS: CD8 T cell $\approx$ escape $\approx \mathrm{HIV}$ - polyfunctional $\approx$ vaccine}

HIV-1 is the cause of one of the most destructive pandemics in human history, with 33.4 million people currently infected worldwide [101]. Development of a vaccine that could prevent acquisition or disease associated with approximately 7000 new infections occurring daily remains an urgent priority in global health. The difficulty in defining consistent and easily measurable biological marker(s) of immune protection has been among the key scientific challenges to HIV vaccine development to date. Defining single genes, cells or molecules as biomarkers is inherently problematic when even the respective roles of antibodies, CD4 T cells, CD8 $\mathrm{T}$ cells or innate arms of the immune response at mucosal and systemic sites are not completely understood. Furthermore, the function of each of these arms in any individual is influenced by host genetic polymorphisms, viral evasion strategies and complex interactions between multiple markers. In general, immunity against human pathogens operates as a coordinated system of many interdependent biological processes and not as a response involving mutually exclusive or independent systems (i.e., purely humoral or purely cellular). Nevertheless, HIV vaccine research has historically differentiated along these lines based on alternative goals of inducing long-lived protection from HIV infection versus achieving control of viral replication in established infection. The typical serological responses against HIV-1, characterized by late production of neutralizing antibody against the autologous virus well after viremia peaks and viral reservoirs are established, does not provide a model of natural immunity that can be used as the basis for developing vaccine sterilizing immunity. Hence, rather than recapitulating the optimal responses found in nature as other successful vaccines have done, a preventative HIV vaccine would have to do better than nature.

Of all infectious diseases that can be successfully prevented by vaccination currently, neutralizing antibodies are a correlate of protective immunity [1]. In the Rhesus macaque model, passive immunization with broadly neutralizing anti-HIV Env monoclonal antibodies protect against simian HIV infection [2]. Although this suggests that induction of such antibodies should be a requirement of active immunization with an HIV-1 Env immunogen, there are a variety of mechanisms that impede the production or efficacy of these antibodies in vivo [3]. Despite these issues, the recent Phase III RV144 HIV vaccine trial buoyed the prospects of ultimately achieving protective humoral responses. The canary pox vector encoding HIV-1 Gag, Env and protease and subtype B/E gp120 protein boost vaccine provided a modest $31 \%$ efficacy against HIV-1 acquisition [4], and preliminary data raise the possibility that the very early presence of subtype B and E gp120-specific binding antibodies, which subsequently waned, played a role in blocking mucosal transmission [3]. Detection of other non-neutralizing antibodies suggests that other viral inhibitory functions of antibodies, such as $\operatorname{IgA}$ and $\operatorname{IgM}$ aggregation of free virions through the mucosa, antibody-dependant cellular cytotoxicity and cell-mediated viral inhibition, may have particular value in being more easily induced, present very early in the immune response and perhaps less subject to viral mutational escape [3].

As the obstacles associated with antibodymediated immunity came to light over time, accumulated data across clinical observational studies, animal model experiments, HIV-1
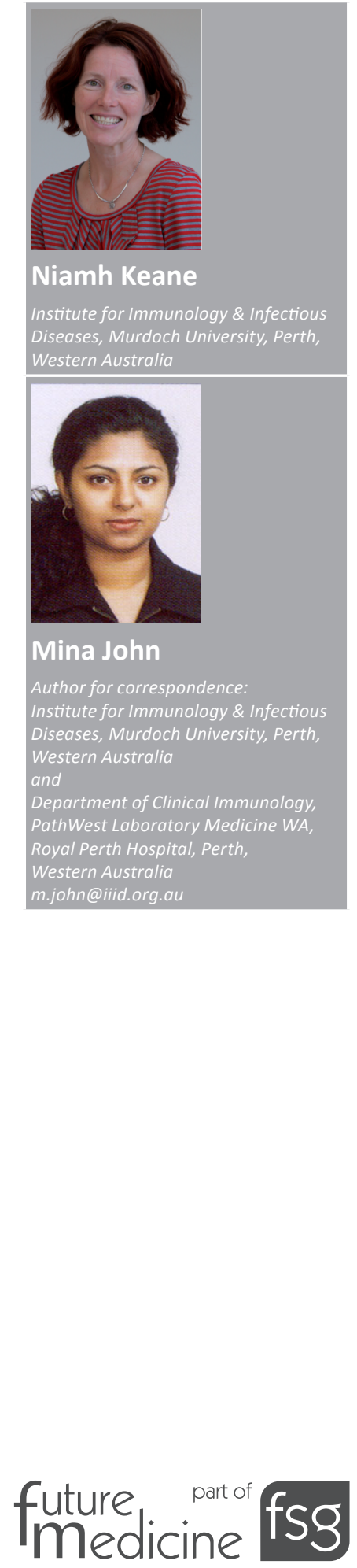
evolution research and host genetic associations revealed the key role of CD8 T cells in viral inhibition in vivo, and this was important in driving many HIV vaccine researchers towards an alternative goal of containment of early viral replication by $\mathrm{T}$ cells and prevention of HIV-associated immune depletion. A natural precedent for this may be found among 'HIV controllers' who maintain low stable virus concentrations, including to below 40 copies $/ \mathrm{ml}$ in the most extreme cases, without any antiretroviral therapy. The first human proof-of-concept study for pure T-cell-based vaccines was the Step trial, using an adenovirus serotype 5 (MRKAd5) vector encoding HIV-1 subtype B Gag, Pol and Nef proteins. There was an unexpected higher rate of HIV-1 acquisition associated with this vaccine, and no difference in viral load set point between vaccinated or unvaccinated infected cases was observed [5]. Studies of IFN- $\gamma$ responses and other intracellular cytokines in trial participants have not provided evidence that the response rate or magnitude of these responses played a protective role against HIV infection or viral control [6]. Although Step and a related MRKAd5 trial were terminated, eliciting strong CD8 T-cell immunity remains a desired outcome in ongoing trials of other vaccine candidates. Even with renewed focus on humoral immunity post-RV144, potent CD8 T-cell responses could play an ancillary role in viral clearance if a HIV-specific antibody is indeed necessary but not sufficient for infection prevention in humans. The evidence for CD8 $\mathrm{T}$ cells in natural infection continues to strengthen. A recent genome-wide association study involving $974 \mathrm{HIV}$ controllers identified that the most significant genetic determinants of $\mathrm{HIV}$ control were all within the MHC and were specifically explained by particular amino acids within the peptide-binding groove of HLA-B alleles, as well as one residue in the transmembrane domain of HLA-C [7]. The refining of genetic associations to residues in the HLA-B peptide-binding groove provide the strongest argument to date for the primacy of the functional interaction between intracellularly processed HIV peptides and HLA-restricted CD8 T cells to effective antiviral cytotoxicity in established infection. These results support previous independent genome-wide association study data that used viral load set point and disease progression as clinical outcomes, and build upon a large body of preceding candidate gene research in the HIV field, which consistently identified the HLA-B locus in general and HLA-B alleles in particular as key genetic biomarkers of immune protection [8]. Another major line of evidence for the importance of CD8 T cells is derived from studies of HIV mutational escape and genetic diversity. The extreme variability of circulating HIV-1 strains is what mandates especially broadbased immunity from an HIV vaccine. A large number of mutational networks in population HIV sequences are HLA class I allele specific, suggesting that there are frequent and predictable patterns of intra-host immune selection by CD8 T-cell responses in vivo [9]. The rapid selection of HIV-1 escape mutations can occur as early as 30 days post-transmission, before antibody seroconversion and is associated with the acute CD8 T-cell response that mediates initial viral suppression to set point levels [10].

\section{"Development of a cohesive and widely available strategy to establish functional profiles of CD8 T cells ... would ... be very useful in the context of HIV immunology and vaccine research."}

The cellular biomarker(s) of an effective HIVspecific CD8 T-cell response has therefore been of major interest. The IFN- $\gamma$ enzyme-linked immunospot (ELISpot) assay has played a dominant role in the CD8 T-cell field. Although IFN- $\gamma$-based immunoassays are amenable to high-throughput testing and are sensitive for detection of epitope-specific responses, the magnitude or breadth of these responses alone do not correlate with plasma viral load or CD4 T-cell count in chronic infection [11]. Vaccinespecific CD8 T-cell IFN- $\gamma$ responses were demonstrated in vaccinees who subsequently acquired progressive infection in the Step trial, suggesting that other qualitative aspects of CD8 $\mathrm{T}$ cells are important [6]. Cellular functions associated with better outcome in HIV-1 infection include dual IFN- $\gamma$ and IL-2 production, degranulation and proliferative capacity (CD8 T-cell polyfunctionality) and upregulation of perforin on stimulation in vitro $[12,13]$. A study of HIV controllers found they had potent percell cytotoxicity mediated by greater loading and delivery of granzyme B to target cells [14], and data from Harari et al. suggests that perforin and granzyme B levels correlate with direct cytotoxic activity in viral peptide-pulsed target cells assayed by chromium release [15]. CD8 memory differentiation (marked by the expression of CCR7, CD45Ra, CD28, CD127, CD27 and PD-1) imposes further variation in cytotoxic granule expression associated with early-, intermediate- and late-stage memory cells. A recent 
study of acute infection found that CD8 T-cell responses were largely oligofunctional, and MIP$1 \beta$-producing CD8 T cells were associated with the earliest escape mutations [16]. Currently, many biomarkers are being studied as separate or partially overlapping subsets. Development of a cohesive and widely available strategy to establish functional profiles of CD8 T cells incorporating phenotype, differentiation/maturation states, as well as cytotoxicity and other noncytotoxic effector functions simultaneously would therefore be very useful in the context of HIV immunology and vaccine research.

Whilst there are many established and emerging biomarkers germane to antibody and T-cell-mediated immune responses, the remarkable adaptability of HIV to these responses means that it is important to interpret qualitative immunological data taking the specificity of the stimulating antigen into account, and the HLA-mediating antigen presentation. For example, certain epitopes in chronic progressive HIV infection appear to be created as a result of CD8 $\mathrm{T}$-cell selection pressure and then elicit high IFN- $\gamma$-producing, highly avid T-cell responses [17]. Hence, for this HLA/epitope context, high magnitude IFN- $\gamma$ expression should not be interpreted as marking a protective immune response and may even be actively viral promoting. Conversely, HLA-B-restricted responses are more polyfunctional [18] and certain protective HLA-B alleles such as $H L A-B^{*} 5701$ were associated with higher magnitude Gagspecific responses in the Step trial [19]. These data suggest that HLA genotype, viral protein and whether a viral epitope used in vitro is actually adapted to a cell response in vivo, should all be considered when assessing the utility of biomarkers of immune protection.

As the relatively newer fields of mucosal immunity and other innate mechanisms of immune defense develop, it is increasingly apparent that, as in nature, a protective vaccine immune response will be the result of synergistic and wellregulated interactions between multiple systems, cells and molecules, and therefore a whole profile or biomarker signature based on multiple characteristics will be superior to single biomarkers considered in isolation. For highly variable adaptable pathogens, such as HIV, all immunological biomarkers must be interpreted with knowledge of the viruses' evolutionary solutions to escape or exploit human immune defences.

\section{Financial \& competing interests disclosure}

The authors have no relevant affiliations or financial involvement with any organization or entity with a financial interest in or financial conflict with the subject matter or materials discussed in the manuscript. This includes employment, consultancies, honoraria, stock ownership or options, expert testimony, grants or patents received or pending, or royalties.

No writing assistance was utilized in the production of this manuscript.

\section{Bibliography}

1 Plotkin SA: Correlates of protection induced by vaccination. Clin. Vaccine Immunol. 17(6), 1055-1065 (2010).

2 Mascola JR, Stiegler G, VanCott TC et al.: Protection of macaques against vaginal transmission of a pathogenic HIV-1/SIV chimeric virus by passive infusion of neutralizing antibodies. Nat. Med. 6(2), 207-210 (2000).

3 Haynes BF, Moody MA, Liao HX, Verkoczy L, Tomaras GD: B cell responses to HIV-1 infection and vaccination: pathways to preventing infection. Trends Mol. Med. 17(2), 108-116 (2011).

4 Rerks-Ngarm S, Pitisuttithum P, Nitayaphan S et al.; MOPH-TAVEG Investigators: ALVAC and AIDSVAX to prevent HIV-1 infection in Thailand. N. Engl. JMed. 361(23), 2209-2220 (2009).

5 Buchbinder SP, Mehrotra DV, Duerr A et al.; Step Study Protocol Team: Efficacy assessment of a cell-mediated immunity
HIV-1 vaccine (the Step Study): a doubleblind, randomised, placebo-controlled, test-of-concept trial. Lancet 372(9653), 1881-1893 (2008).

6 McElrath MJ, De Rosa SC, Moodie Z et al.; Step Study Protocol Team: HIV-1 vaccineinduced immunity in the test-of-concept Step study: a case-cohort analysis. Lancet 372(9653), 1894-1905 (2008).

7 Pereyra F, Jia X, McLaren PJ, Telenti A, de Bakker PI, Walker BD; International HIV Controllers Study: The major genetic determinants of HIV-1 control affect HLA class I peptide presentation. Science 330(6010), 1551-1557 (2010).

8 Fellay J: Host genome influences on HIV-1 disease. Antivir. Ther. 14(6), 731-738 (2009).

9 Brumme Z, John M, Carlson JM et al.: HLA-associated immune escape pathways in HIV-1 subtype B Gag, Pol and Nef proteins. PLOS One 4(8), e6687 (2009).

10 Goonetilke N, Liu MK, Salazar-Gonzalez JF et al.: The first $\mathrm{T}$ cell response to transmitted/founder virus contributes to the control of acute viremia in HIV-1 infection. J. Exp. Med. 206(6), 1253-1272 (2009).

11 Addo MM, Yu XG, Rathod A et al.: Comprehensive epitope analysis of human immunodeficiency virus type-1 (HIV-1)specific T-cell responses directed against the entire expressed HIV-1 genome demonstrate broadly directed responses, but no correlation to viral load. J. Virol. 77(3), 2081-2092 (2003).

12 Betts MR, Nason MC, West SM et al:: HIV nonprogressors preferentially maintain highly functional HIV specific CD ${ }^{+}$T cells. Blood 107, 4781-4789 (2006).

13 Hersperger AR, Pereyra F, Nason M et al.: Perforin expression directly ex vivo by $\mathrm{HIV}$-specific CD8 T-cells is a correlate of HIV elite control. PLoS Pathog. 6(5), e1000917 (2010).

14 Migueles SA, Osborne CM, Royce C et al.: Lytic granule loading of CD8 ${ }^{+} \mathrm{T}$ cells is required for $\mathrm{HIV}$-infected cell elimination associated with immune control. Immunity 29(6), 1009-1021 (2008). 
15 Harari A, Enders FB, Cellerai C, Bart PA, Pantaleo G: Distinct profiles of cytotoxic granules in memory CD8 T cells correlate with function, differentiation stage, and antigen exposure. J. Virol. 83(7), 2862-2871 (2009).

16 Ferrari G, Korber B, Goonetilleke N et al.: Relationship between functional profile of HIV-1 specific CD8 T cells and epitope variability with the selection of escape mutants in acute HIV-1 Infection. PLoS Pathog. 7(2), e1001273 (2011).
17 Keane N, Roberts S, Laird R et al: Selection of higher avidity HLA-restricted $\mathrm{T}$ cell responses as a viral adaptationstrategy. Presented at: AIDS Vaccine. Paris, France, 19-22 October 2009.

18 Harari A, Cellerai C, Enders FB et al.: Skewed association of polyfunctional antigen-specific $\mathrm{CD} 8 \mathrm{~T}$ cell populations with HLA-B genotype. Proc. Natl Acad. Sci. USA 104(41), 16233-16238 (2007).
19 Fellay J, Frahm N, Shianna KV et al.: Host genetic determinants of $\mathrm{T}$ cell responses to the MRKAd5 HIV-1 gag/ $\mathrm{pol} / \mathrm{nef}$ vaccine in the Step trial. J. Infect. Dis. 203(6), 773-779 (2011).

\section{Website}

101 Global report: UNAIDS report on the global AIDS epidemic 2010 www.unaids.org/en/resources/ unaidspublications/2010/ 